\title{
Extrusión de Perfiles Espumados de Madera Plástica
}

\author{
Oscar Francisco Delgado*, Jorge Alberto Medina**.
}

\begin{abstract}
Plastic / natural fiber composite foams offer the advantages of both the composite and foamed materials. In this research, the effects of the processing conditions and the concentration and type of CFA (chemical foaming agent) on the physical and morphological parameters of foamed HDPE / coffee husk composites were studied, with the objective of achieve continuous production of these materials in extrusion, using a CFA. Each step of the process is addressed, including the selection of materials, mixing, pelletization, and extrusion - foaming of the composite, and the characterization of relevant physical and morphological parameters of the obtained product. The greatest potential for this materials, is in building products that have limited structural requirements offering lower maintenance, high durability and lower environmental impact compared with the use of solid wood.
\end{abstract}

Palabras Claves: Extrusión, Materiales compuestos, Madera plástica, Procesos de espumado, Agentes espumantes químicos.

\section{INTRODUCCIÓN}

En el CIPP, Centro de Investigación en Procesamiento de Polímeros de la Universidad de los Andes, se ha buscado implementar procesos productivos que aprovechen la gran cantidad de residuos orgánicos que produce la industria agrícola y maderera en Colombia, usándolos como refuerzo en materiales plásticos, con el fin de reemplazar a la madera en algunas de sus aplicaciones. Estos materiales compuestos reciben el nombre de compuestos de madera plástica o WPC por su sigla en ingles (Wood Polymer Composites).
El mercado actual para estos compuestos solo en Norteamérica alcanzó ya 600.000 toneladas anuales. Actualmente, la fibra de madera es el material más utilizado como refuerzo. Se estima que la extrusión de materiales plásticos con rellenos y refuerzos de fibras naturales registrará un crecimiento anual de más del 10\% anual durante los próximos cinco años [2]. El rápido crecimiento que viene presentando este tipo de materiales se explica mediante las ventajas que estos ofrecen en términos de costos, durabilidad e impacto ambiental. Algunas de estas ventajas son:

\footnotetext{
* Estudiante Maestría Ing. Mecánica, Universidad de los Andes, Bogotá, Colombia. e-mail: o-delgad@uniandes.edu.co

** Profesor asociado, Director del Centro de Investigació en procesamiento de polímeros, CIPP, Ing. Investigador del Dpto. de Ing. Mecánica, Universidad de los Andes, Bogotá, Colombia. e-mail: jmedina@uniandes.edu.co
} 
- Abundancia y bajo costo de la materia prima. Los materiales plásticos utilizados en este tipo de compuestos pueden provenir de un proceso de reciclaje, lo cual disminuye su costo. Por otro lado las fibras naturales que se utilizan como refuerzo constituyen un desperdicio de la industria agrícola.

- Bajo impacto ambiental. La utilización de este tipo de materiales promueve el reciclaje, aprovecha un desecho agroindustrial, hace uso de materiales biodegradables (en el caso de las fibras naturales) y evita la tala de bosques en la medida en que estos materiales se utilizan como reemplazo de la madera en algunas de sus aplicaciones.

- Baja densidad. La baja densidad, característica primordial de los productos espumados, permite diseñar componentes ligeros y de buena rigidez como los paneles tipo sándwich o estructuras en coextrusión. Muchos perfiles de madera plástica espumada son coextruidos con una capa no espumada exterior sobre un corazón espumado, ahorrando costos y disminuyendo peso sin sacrificar en mayor medida las capacidades estructurales del perfil.

- Apariencia y procesabilidad de la madera. La superficie de los productos de madera plástica se puede tratar como la madera: se le puede pegar un acabado en chapa, recubrir con barnices, clavar, atornillar, taladrar, cepillar. También se pueden formar artículos similares a los encontrados con los productos plásticos tradicionales.

- Durabilidad. Los compuestos de madera plástica requieren menor mantenimiento que las maderas comunes, ya que no se ven afectados ni por la humedad ni por los insectos. Lo anterior los hace apropiados para aplicaciones en las cuales los productos se someten a condiciones ambientales severas.

- Bajo nivel de abrasión de las fibras. Las fibras naturales poseen la ventaja de ser menos abrasivas que otros tipos de refuerzo basados en componentes minerales, promoviendo mayor duración de los equipos.

- Rigidez y estabilidad dimensional. Estas dos propiedades se verán incrementadas median- te la adición del refuerzo, siempre y cuando se promueva una buena adhesión en la interfaz matriz / refuerzo.

Esta investigación se basa en la producción continua de perfiles espumados de madera plástica en extrusión mediante el uso de agentes espumantes químicos, basados en materiales de fácil adquisición y disponibilidad en el entorno colombiano. También se busca establecer los principales parámetros de proceso que puedan incidir en las características del producto final.

Se seleccionó el Polietileno de Alta Densidad (PEAD) grado soplado para servir de matriz polimérica. Este polímero se caracteriza por su resistencia química y sus capacidades como aislante eléctrico, su tenacidad y su relativamente bajo coeficiente de fricción. Se utiliza generalmente en empaques, botellas flexibles y contenedores de productos químicos, garantizándose un volumen considerable de material potencialmente reciclable. El Polietileno además de ser el plástico de mayor producción y consumo en el mundo, es uno de los más baratos. El PEAD grado soplado, presenta mayor viscosidad y mayor resistencia de masa fundida lo cual facilita la generación y control en el desarrollo de espumas.

Se seleccionó el cisco o cascarilla de café como materia prima para servir de refuerzo en el material compuesto. La selección se realizó principalmente por la alta oferta de esta fibra vegetal en el entorno colombiano y porque sus características físicas y químicas [l] se adaptan correctamente a las necesidades del proceso. Esta selección se llevó a cabo teniendo en cuenta que la utilización masiva de esta fibra en materiales compuestos en Colombia, puede representar una solución al problema ambiental que generan actualmente dichos desperdicios, representando además, una fuente potencial de ingresos adicional para los caficultores nacionales.

Se utilizó Anhídrido Maléico (AM) como agente de acople para promover la adhesión entre 
las fibras hidrofílicas del cisco de café y el PEAD de naturaleza hidrofóbica. El AM fue seleccionado en base a investigaciones recientes llevadas a cabo en el CIPP [3] [4].

Los agentes espumantes químicos, son compuestos que se descomponen en rangos de temperatura bien definidos, liberando grandes cantidades de gas (en este caso, dióxido de carbono). Por su comportamiento térmico ante el inicio de su descomposición, los agentes espumantes químicos se dividen en agentes exotérmicos o endotérmicos. Como agente espumante, se utilizaron dos formulaciones de Hydrocerol ${ }^{\grave{ }}$. Estas fueron seleccionadas de manera que sus temperaturas de proceso coincidieran con las temperaturas de proceso del PEAD y no sobrepasaran la temperatura de inicio de descomposición térmica del cisco de café.

\section{EXPERIMENTACIÓN}

\section{Materiales}

Como matriz, se utilizó PEAD grado soplado, reciclado y granulado, de $0.929 \mathrm{~g} / \mathrm{cm}^{3}$ de densidad y un Indice de fluidez de $0.346 \mathrm{~g} / 10 \mathrm{~min}$ bajo condición $190^{\circ} ð \mathrm{C} / 2.16 \mathrm{~kg}$ según la caracterización realizada.

El refuerzo seleccionado fue cisco de café, proveniente de cultivos en el departamento del Huila, este fue conseguido a través de la trilladora Cundicafé. La distribución del tamaño de partícula muestra que más del $90 \%$ de las fibras utilizadas poseían tamaños menores a 300 micrómetros.

Para lograr la compatibilidad entre la matriz y la fibra, se utilizó como agente de acople un PE lineal con adición de 8\% Anhídrido Maléico (PE maleato) el cual corresponde a la referencia Cel-Span 334 de Phoenix Plastics.

Para el proceso de espumación química, se

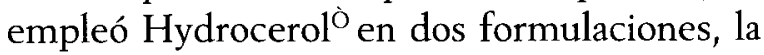
primera, Hydrocerol CT1502 corresponde a una combinación de agentes espumantes químicos exotérmicos y endotérmicos diseñado para extrusión de láminas y perfiles para poliolefinas incluyendo maderas plásticas. La segunda, Hydrocerol CT1619 corresponde a un agente espumante químico endotérmico con propósitos semejantes [1].

\section{Implementación del Proceso}

\section{Preparación y caracterización de materiales}

Se caracterizó la distribución de tamaño de partícula para el cisco de café, así como la densidad y el índice de fluidez para el PEAD, lo anterior con el fin de asegurar que las características de la materia prima se ajustan a los requerimientos del proceso. Se realizaron pruebas de calorimetría de barrido diferencial DSC (Differential Scanning Calorimetry) a una tasa de $10^{\circ} \mathrm{C} / \mathrm{min}$, para establecer claramente las temperaturas de inicio de reacción, las tasas de descomposición y las temperaturas óptimas de proceso de las dos referencias de Hydrocerol. Para adecuarse a las condiciones del proceso, el cisco de café se sometió a procesos de tamizado y secado [1].

Una vez realizada la preparación y caracterización de los materiales, se siguieron los siguientes pasos:

\section{Granulado de madera plástica.}

Para el proceso de granulado se utilizó una extrusora monotornillo marca MPM con motor de 7HP, tornillo de 1.5 pulgadas de diámetro y relación $L / D$ de $24: 1$. Se conectó a una granuladora marca GALA modelo LPU con capacidad para procesar $70 \mathrm{~kg} / \mathrm{hr}$. Esta posee una cuchilla giratoria sumergida en un flujo de agua, lo cual permite corte y enfriamiento del material al mismo tiempo.

Se implementó el proceso de granulado basados en investigaciones anteriores [3] [4], con el fin de obtener el compuesto de madera plástica que sirvió de materia prima para el poste- 
rior proceso de extrusión de espumas. El proceso se ve esquematizado en la figura No.l.

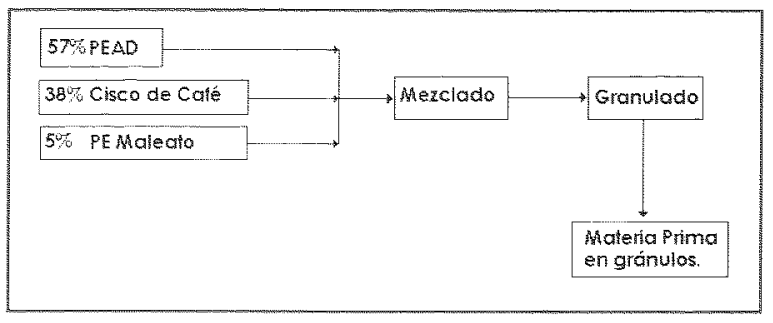

Figura 1. Proceso de gramulado de compuesto de madera plástica

Se utilizó cisco de café en una proporción de $40 \%$ en peso con respecto al polímero y polietileno maleato en un $5 \%$ en peso con respecto a la mezcla. Se mezclaron los componentes en un sistema de tamboreo durante $15 \mathrm{mi-}$ nutos, para asegurar la obtención de una mezcla homogénea. Posteriormente se extruyó el material a la velocidad de tornillo mas alta posible, para asegurar una buena dispersión y mezcla de las fibras de café dentro de la matriz, y así obtener un producto más homogéneo. En la figura No.2 se aprecia la materia granulada obtenida.

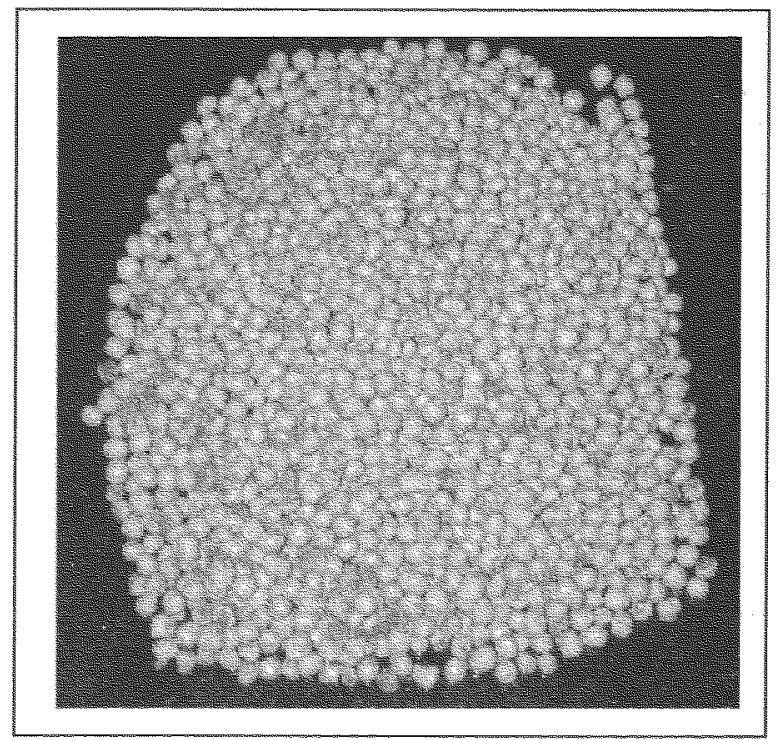

Figura 2. Gránulos de madera plástica

\section{Extrusión de espumas de madera plástica}

Se trabajó en una extrusora monotornillo Brabender Plasticorder modelo 2523 con un tornillo de diámetro nominal de $3 / 4$ de pulgada y una relación longitud a diámetro de 25/1. Los puertos de venteo fueron cerrados para evitar la pérdida de gas a través de los mismos. Se utilizó un dado de perfil diametral de 9.8 mam.

El proceso incluye fusión y mezcla de las materias primas para producir una masa fundida uniforme. Se extruye a una temperatura apropiada que produzca descomposición completa del CBA dentro del barril de la máquina. La masa a espumar debe mantenerse bajo suficiente presión para mantener el agente espumante en solución con el polímero y luego ser extruido a través del dado para permitir la espumación. El proceso se ve esquematizado en la figura No. 3.

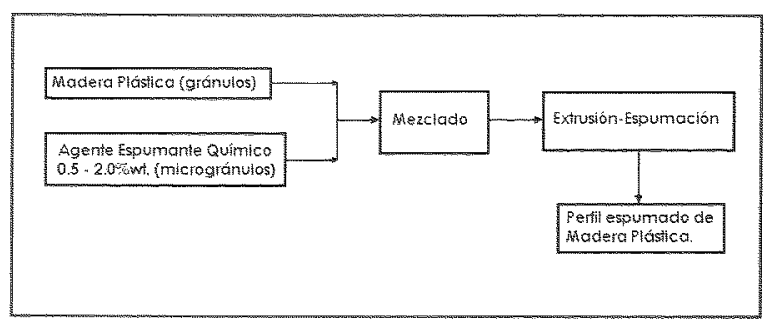

Figura 3. Proceso de extrusión-espumación de maderas plásticas

\section{Perfil de Temperatura:}

El perfil de temperatura a lo largo del tornillo es determinante para la obtención de espumas de buena calidad. La temperatura en la zona de alimentación debe ser baja para prevenir descomposición prematura del agente espumante y posible pérdida de gas a través de la tolva. La temperatura en la zona de compresión debe asegurar plastificación del material, sin promover tasas de reacción altas para el agente espumante. Para una correcta y completa liberación del gas aportado por el agente espumante, las temperaturas equivalentes a los picos de liberación de gas deben lograrse en la zona de dosificación del tornillo. Refiérase a [1] para obtener mayor información acerca del perfil utilizado.

\section{Ventana Operativa}

La ventana operativa, es el rango de temperaturas, presiones y velocidades de tornillo que 
permiten la operación estable de la extrusora y la obtención de una viscosidad apropiada para garantizar la obtención de una estructura espumada homogénea e integra.

Se usó un valor de $1.5 \%$ en peso de agente espumante como límite máximo en la experimentación, al observar que utilizando una composición de $2.0 \%$ en peso, la generación de gas era muy alta tornándose incontrolable el proceso.

El proceso experimental para hallar la ventana operativa, se hizo mediante variaciones de $5^{\circ}$ ðC en la temperatura del dado, partiendo de $120^{\circ} \partial \mathrm{C}$ hasta llegar a $145^{\circ} \partial \mathrm{C}$. La velocidad de rotación del tornillo se varío comenzando en 0 RPM hasta lograr una operación estable.

\section{Obtención de muestras.}

Se obtuvieron perfiles de madera plástica utilizando las dos referencias de Hydrocerol, variando la concentración de agente espumante y la temperatura del dado como indica la figura No.4 [1]. Luego de obtenidas las muestras, se realizaron pruebas de densidad, microscopía y compresión para evaluar las propiedades obtenidas y correlacionarlas con los parámetros variados.

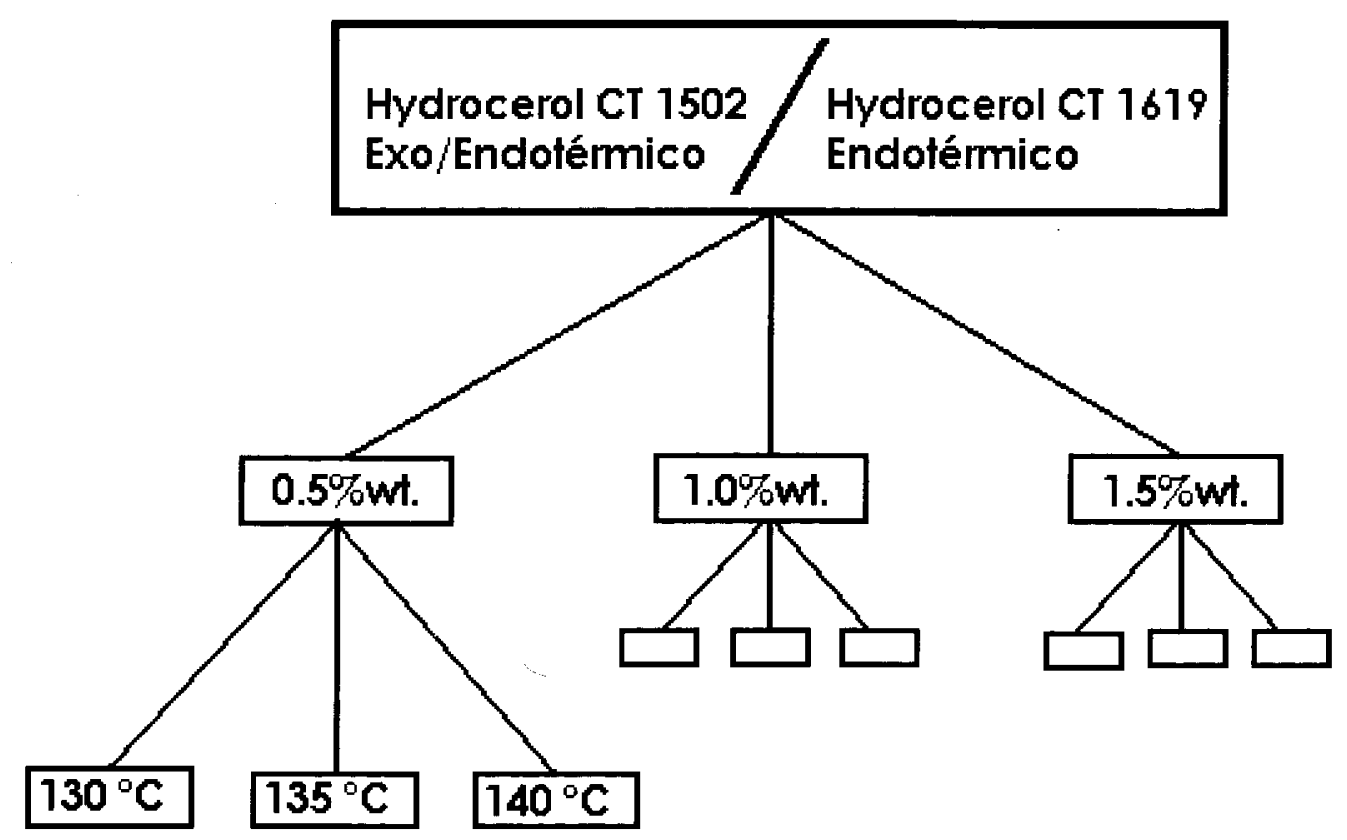

Figura 4. Diagrama de Experimentación.

\section{Resultados y Análisis}

La ventana operativa para ambos agentes espumantes resultó ser similar. A temperaturas del dado menores a $130^{\circ} \partial \mathrm{C}$, se presentaba sobreespumación, impidiendo la estabilización del extrudido sin importar la velocidad del tornillo manejada. A temperaturas del dado mayores a $140^{\circ} \partial \mathrm{C}$ la viscosidad de la masa fundi- da era tan baja que se producían fluctuaciones y muy baja espumación por perdida del gas a través de la masa fundida.

Por lo tanto, la ventana operativa para este proceso incluye el rango de temperaturas del dado entre $130^{\circ} \mathrm{C}$ y $140^{\circ} \mathrm{C}$, velocidades entre $20 \mathrm{rpm}$ y $40 \mathrm{rpm}$ y concentraciones de agente espumante entre $0.5 \%$ y $1.5 \%$. 


\section{Prueba de Densidad}

Se realizó la prueba de densidad según norma ASTM D792 a los perfiles obtenidos. En la figura No.5 Se muestra el efecto de la concentración de agente espumante y temperatura de extrusión en la razón de expansión del compuesto espumado (densidad del sólido / densidad de la espuma).

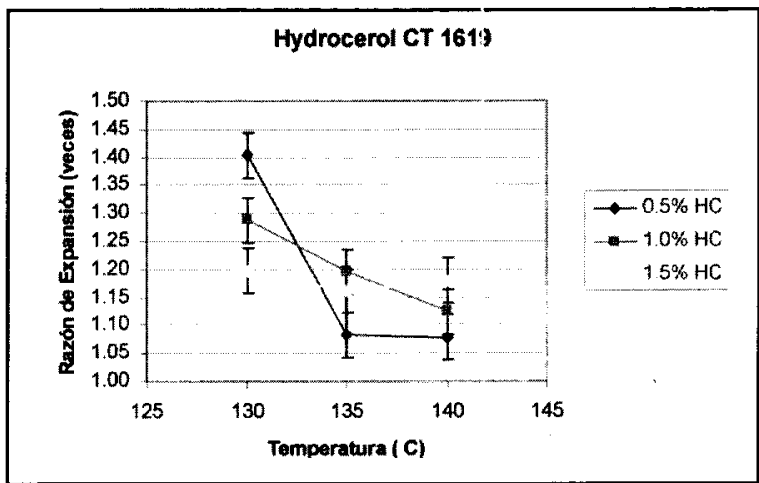

Figura 5. Efecto de la concentración de agente espumante y temperatura de extrusión en la razón de expansión del compuesto espumado.

Se puede apreciar que las mayores razones de expansión corresponden a bajas temperaturas del dado. Esto se debe a que existe mayor viscosidad de la masa fundida y mayor contrapresión, lo que permite una mayor resistencia de la masa, mayor solubilidad del gas en la matriz y por lo tanto mayor nivel de espumación.

También se observa que no es necesaria la presencia de gran cantidad de agente para obtener buenas razones de expansión. De hecho se lograron mayores razones de expansión con menores concentraciones de agente espumante. El exceso de gas, además de su efecto lubricante (baja la viscosidad y resistencia de la masa fundida), genera un nivel de presión que promueve sitios de escape para el gas, haciendo poco eficiente la labor del agente espumante. Se nota también que la sensibilidad de la razón de expansión a los cambios en temperatura disminuye a medida que se aumenta la concentración de agente espumante.
A la temperatura de $140^{\circ} \mathrm{C}$ no parece haber mayor diferencia entre las muestras a distintas concentraciones de agente espumante. $\mathrm{Al}$ parecer, a esta temperatura la masa fundida es tan poco viscosa que impide una buena espumación. Se observa un fenómeno conocido como límite de contención de gas, en donde el tamaño de celda y la densidad permanecen insensibles al contenido o al tipo de agente espumante.

La explicación al límite de contención de gas, es que una vez las celdas han sido nucleadas, continúan su crecimiento mientras exista suficiente difusión de gas dentro de ellas. Una vez alcanzan el mayor tamaño limitado por la resistencia de la masa fundida, ocurre coalescencia y ruptura de las celdas si se incrementa el nivel de gas, disminuyendo el tamaño de celda promedio e impidiendo una mejor reducción de densidad [8].

\section{Microscopía:}

Con el fin de obtener información sobre la estructura celular obtenida, se examinaron las muestras obtenidas en el estereoscopio (40X). Se estudió la superficie de fractura de las muestras indagando sobre el tamaño promedio y la forma predominante de las celdas.

La cuantificación del tamaño promedio de celda se estimó mediante la medición del diámetro nominal de celda a lo largo de una línea recta imaginaria recorriendo el perfil desde su punto medio y calculando el valor promedio correspondiente.

El número de celdas por unidad de volumen de espuma (Nc) es un parámetro importante, usado en la estimación de la efectividad de un agente nucleante. Es una función del tamaño de celda y la densidad de la espuma plástica así [7].

$$
N_{C} \cong \frac{1-P / P_{P}}{10^{-4}}
$$


Donde:

Nc es el número de celdas por $\mathrm{cm}^{3}$. (densidad de población celular)

p es la densidad de la espuma en $\mathrm{gr} / \mathrm{cm}^{3}$.

$\rho_{\mathrm{p}}$ es la densidad del material sólido espumado en $\mathrm{gr} / \mathrm{cm}^{3}$.

$d$ es el diámetro promedio de celda en $\mathrm{mm}$.

Los resultados mostraron que la densidad de población de celdas es mayor cuando se han logrado mayores reducciones de densidad. A mayor efectividad del agente nucleante, es decir, a mayor generación de celdas, habrá mayores reducciones de densidad.

También se indagó sobre el efecto de la concentración de agente espumante y temperatura de proceso en el tamaño promedio de celda. Los menores tamaños de celda, los cuales favorecen las propiedades mecánicas del producto espumado, se dieron en los casos en los cuales el nivel de espumación fue bajo.

Lo anterior se explica porque las grandes reducciones de densidad, se logran cuando existe tiempo suficiente para el crecimiento de celdas, en este caso no se están generando nuevas celdas sino que las celdas presentes sufren un proceso de crecimiento generando una estructura de celdas gruesas. Cuando no se logra tanta reducción de densidad, las celdas no tienen tanto tiempo de crecer y permanece una estructura de celdas finas.

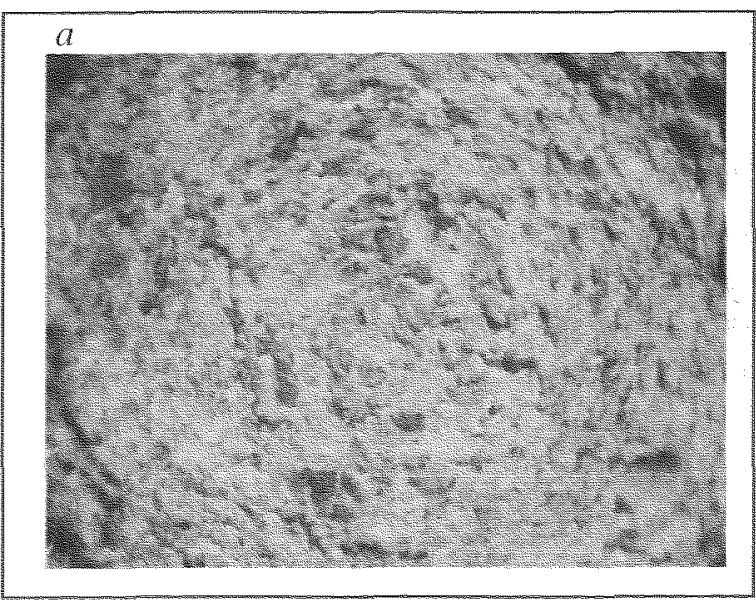

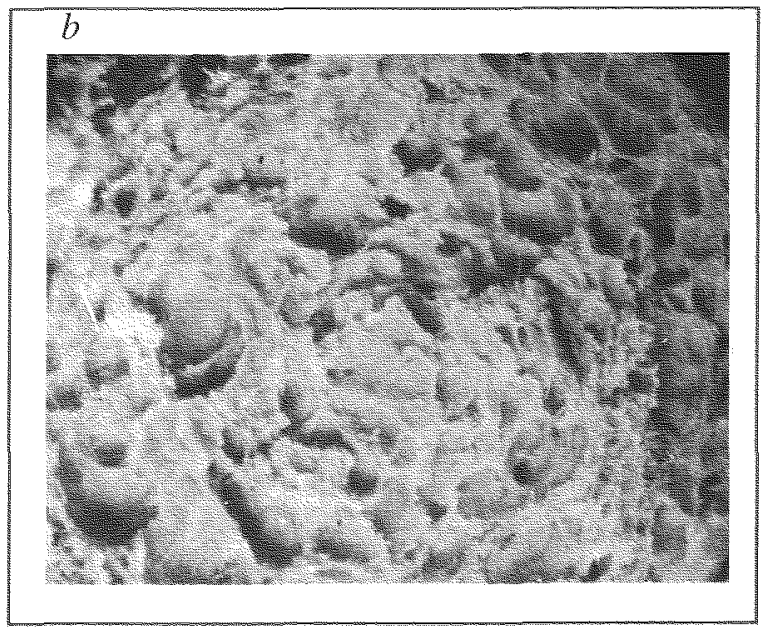

Figura 6. Comparación de estructuras celulares fina (a) y gruesa (b) a un aumento de $40 \mathrm{X}$.

En la figura No.6, podemos ver microfotografías en donde encontramos una comparación entre las estructuras celulares fina y gruesa obtenidas.

La forma predominante de las celdas fue estudiada cualitativamente, observando que al presentarse estructura fina, las células tienden a ser de forma elíptica u ovoide, mientras que en las estructuras gruesas se observa una degeneración de la estructura y se ven predominantemente celdas de formas poligonales irregulares (ver Figura No.6).

\section{Propiedades Mecánicas}

Se obtuvo un producto barato, liviano, con la apariencia de la madera, insensible a la humedad y a los insectos y con la resistencia suficiente para ser utilizado en aplicaciones semiestructurales, decorativas o como relleno espumado en aplicaciones de coextrusión. Se observa que la rigidez del material disminuyó cuando se lograron menores densidades del producto espumado. Se hace necesario entonces, lograr un equilibrio apropiado entre la reducción de peso y costo del producto con las propiedades mecánicas del mismo, ya que mejores propiedades implican mayores densidades (y costos) y menores densidades implican un sacrificio en términos de las propiedades mecánicas. 


\begin{tabular}{|l|l|}
\hline \multicolumn{2}{|c|}{ Propiedades de la Madera Plástica Espumada } \\
\hline Densidad (gr/cc) & $0.51-0.86$ \\
\hline Nc (celdas/cC) & $8500-30000$ \\
\hline Módulo elástico (MPa) & $70-110$ \\
\hline R. a Compresión & $3.6-6.9$ \\
\hline
\end{tabular}

\section{Conclusiones}

La investigación condujo a la implementación de un proceso a escala de laboratorio para generar perfiles espumados de compuestos de madera plástica. Entre las conclusiones más importantes resultado de esta investigación se encuentran:

- La ventana operativa para el proceso y las composiciones utilizadas corresponde a temperaturas de extrusión entre $130^{\circ}$ ðC y $140^{\circ} \partial \mathrm{C}$ y velocidades de tornillo que oscilan entre 20RPM y 40RPM. El contenido óptimo de CFA se encuentra entre 0.5 y $1 \%$ en peso.

- Se logró tener control sobre el proceso y correlaciones entre los parámetros de proceso y las propiedades obtenidas. Lo anterior facilitará la implementación del proceso a una escala productiva.

- Los niveles de espumación mayores correspondieron a menores concentraciones de agente espumante. Utilizar cantidades mayores de agente espumante que las recomendadas dentro de la ventana operativa, encarecerá el proceso y generará problemas como excesiva dislocación de la superficie, tiempos extendidos de ciclo y problemas de control dimensional.

- A temperaturas superiores a $140^{\circ} \partial \mathrm{C}$, se observa un límite de contención de gas en donde el tamaño de celda y la densidad permanecen insensibles al contenido o al tipo de agente espumante.

\section{Referencias}

[1] “Diseño de proceso en la extrusión de perfiles espumados con matriz termoplástica y refuerzo lignocelulósico". Oscar Francisco Delgado Neira. Universidad de los Andes, 2003.

[2] "Plásticos reforzados al natural" Carlos Serrano Mayo 2002

[3] "Materiales compuestos con matriz termoplástica y refuerzo lignocelulósico". Diego Iván García. Universidad de los Andes, 2002.

[4] "Desarrollo de materiales compuestos termoplásticos con componentes naturales". Carlos Andrés Rodríguez. Universidad de los Andes, 2000.

[5] "Mechanical properties of wood flake - polyethylene composites. Part I: effects of processing methods and matrix melt flow behaviour" P.W. Balasuriya, L. Ye, Y.N. Mai.

[6] "Fine celled foaming of plastic / wood-fiber composites" C.B. Park, G.M. Rizvi, H. Zhang.

[7] "Handbook of polymeric foams and foam technology" D. Klempner, K.C. Frisch. Hanser New York (1991).

[8] "Foam extrusion of HDPE / Wood Flour composites using chemical foaming agents". Qingxiu Li, Laurent Matuana.

[9] "Wood plastic composites in the United States, the interfacing of two industries" Craig Clemons.

[10] "Foaming expands possibilities for wood fiber composites" Jan H. Schut

www.plasticstechnology.com/articles/200107fa2.html 